\title{
Sistema modular en bambú guadua con conexiones metálicas de fácil armado para vivienda rural
}

\section{Modular system in guadua bamboo with easy to be assembled metal connections for rural housing}

\author{
Kevin Andrés Casallas Benitez (1) \\ Juan Camilo Sánchez Gutiérrez (1) \\ Caori Patricia Takeuchi (1) \\ (1) Universidad Nacional de Colombia \\ sede Bogotá. \\ kacasallasb@unal.edu.co \\ juacsanchezgut@unal.edu.co \\ cptakeuchit@unal.edu.co
}

Recibido: 16 de Julio, 2019

Aceptado: 10 de Diciembre, 2019

Doi: http://dx.doi.org/10.24133/ciencia. v22i1.1303

"THIS IS AN OPEN ACCESS ARTICLE UNDER THE TERMS OF THE CREATIVE COMMONS ATTRIBUTION LICENSE, WHICH PERMITS USE, DISTRIBUTION AND REPRODUCTION IN ANY MEDIUM, PROVIDED THE ORIGINAL WORK IS PROPERLY CITED."

\begin{abstract}
RESUMEN
En respuesta a la coyuntura que vive Colombia debido a la implementación del acuerdo de paz firmado entre el gobierno y la guerrilla FARC-EP, se hace necesaria una solución habitacional para los nuevos pobladores de las regiones donde se desarrollaba el conflicto armado, que no puede estar fuera del contexto social, ecológico y económico. Es por esto que el bambú Guadua al ser un recurso natural, de fácil acceso y mínimo impacto se convierte en material viable para responder a este problema. Con el uso de conexiones metálicas prefabricadas se agiliza la construcción de viviendas rurales con elementos en guadua, además permitiendo a la estructura cambiar su configuración espacial, aumentando el número de módulos y así la cantidad de espacios habitacionales, según lo permitan las condiciones económicas de sus habitantes, realizando así una construcción progresiva.
\end{abstract}

En el presente documento se muestran los resultados obtenidos en el ensayo de módulos cúbicos de guadua con conexiones en cubos metálicos y su aplicabilidad para la construcción de viviendas de 1 y 2 pisos.

\section{Palabras Claves:}

Bambú, Guadua, Estructuras en bambú, conexión viga-columna, bambú estructural.

\begin{abstract}
In response to the situation that Colombia is experiencing due to the implementation of the peace agreement signed between the government and the FARC-EP guerrillas, a housing solution is necessary for the new inhabitants of the regions where the armed conflict was taking place, which cannot Be out of social, ecological and economic context. This is why Guadua bamboo, being a natural resource, easily accessible and minimal impact, becomes a viable
\end{abstract}


material to respond to this problem. With the use of prefabricated metal connections, the construction of rural houses with elements in guadua is expedited, also allowing the structure to change its spatial configuration, increasing the number of modules and thus the amount of residential spaces, as permitted by the economic conditions of its inhabitants, thus carrying out a progressive construction.

This document shows the results obtained in the test of cubic modules of guadua with connections in metal cubes and its applicability for the construction of houses of 1 and 2 floors.

\section{Keywords:}

Bamboo, Guadua, Bamboo structures, Beam and column joints, structural bamboo

\section{INTRODUCCIÓN}

Para dar una solución al problema que se avecina con la firma de un "acuerdo de paz", de dar vivienda digna y económica a miles de familias que volverán al campo, es necesario realizar viviendas con estructuras de fácil construcción y con materiales que se encuentren en las regiones, además, de garantizar que sean ecológicamente sustentables. La guadua ha demostrado su versatilidad en el uso como elemento estructural (Herrera \& Takeuchi, 2009), de allí la necesidad de aprovechar este recurso.

La Guadua angustifolia Kunth (en adelante Guadua) es la especie de bambú más popular en Colombia, la que más se ha estudiado y la que mejor resultados tiene en pruebas de carga para uso como elemento estructural (Luna, Lozano, \& Takeuchi, 2014), este se usa con popularidad en la región Cafetera y el Valle del Cauca (Colombia), principalmente para las estructuras agropecuarias y viviendas de 1 piso (Osorio et al., 2010).

Las conexiones entre elementos de guadua son objeto de estudio habitual debido a las múltiples formas en las que se pueden realizar las uniones, pero también a lo difíciles que pueden llegar a ser. Varios investigadores (Lamus, Plazas, \& Luna, 2015; Takeuchi, 2004) han realizado ensayos de uniones con perno pasante en elementos de guadua, identificando que, las tensiones perpendiculares a las fibras, son uno de los grandes problemas, ya que la guadua es un material anisótropo con baja resistencia a la tracción perpendicular a las fibras (Takeuchi, 2004).

Los arquitectos Barnet y Jabrane (2019) implementaron una unión para los elementos en bambú, el cual se conecta desde la parte interna del culmo con un elemento tipo cono prefabricado, el cual elimina las perforaciones en la pared o aplicar cemento en los canutos, este tipo de unión de acuerdo a los datos ilustrados llega a una tensión máxima de $1750 \mathrm{~kg}$.

Widyowijatnoko (2012) ha desarrollado una conexión que no utiliza mortero dentro del culmo, sino que usa un elemento flexible tipo cable que lo abraza, mientras atraviesa un perno que es sometido a tensión desde el interior, solucionando así el problema de las tensiones perpendiculares a las fibras y permitiendo una conexión de fácil y rápido armado. Este tipo de conexión es la más fácil de realizar, pues todos los elementos necesarios son de fácil adquisición.

\section{CONEXIONES}

Con el fin de desarrollar un tipo de conexión de fácil armado y que permita la reconfiguración de los módulos para las viviendas, se partió de la investigación de Andry Widyowijatnoko (2012), quien ha realizado numerosos ensayos en diferentes tipos de conexiones donde se pasa un cable de acero dentro de la guadua y un perno de ojo cerrado, para transmitir las cargas del perno a la guadua y viceversa. 


\subsection{ENSAYO DE CONEXIONES}

Se realizaron ensayos de las conexiones de los elementos en guadua como las utilizo en su tesis doctoral Widyowijatnoko (2012), para validar cuales eran las mejores formas de amarre y los elementos más óptimos que se pueden encontrar en el mercado colombiano.

En este ensayo se usaron pernos de ojo de $1 / 2$ " que se encuentran en el mercado cerrando el ojo con soldadura MIG (ver figura 1) para garantizar que no se abriera frente a cargas de tensión, este perno fue enhebrado con cable de acero de diámetro 1/4" y se utilizó la configuración de amarre propuesta por Widyowijatnoko (2012) que se observa en la figura 2.

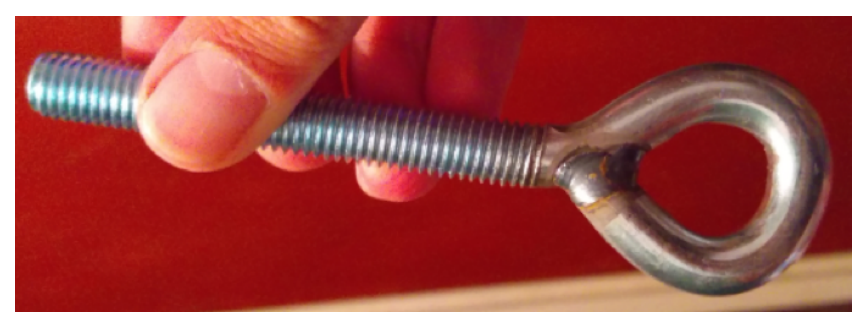

Figura 1. Cierre ojo perno.

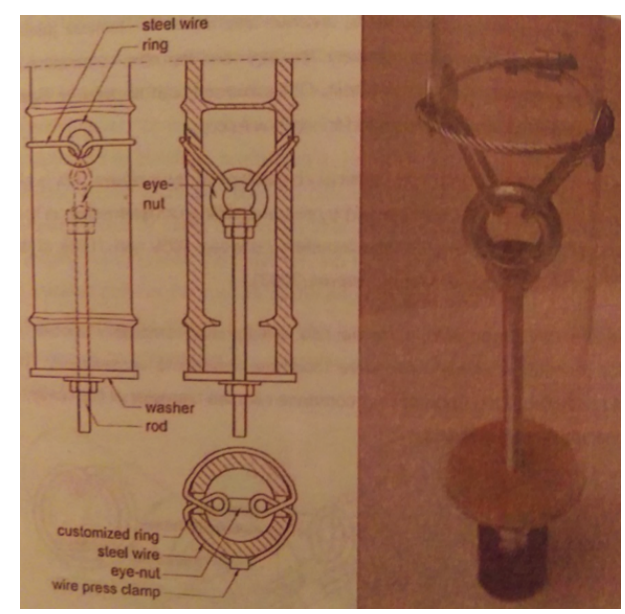

Figura 2. Amarre propuesto por (Widyowijatnoko, 2012)

En las conexiones se amarraron los cables de acero con cuatro pernos, teniendo en cuenta lo que indica la norma NTP 155: cables de acero (Sabate, 1998), además se colocaron ojales metálicos en las perforaciones de la guadua (ver figura 3) para distribuir los esfuerzos en las paredes internas de la perforación.

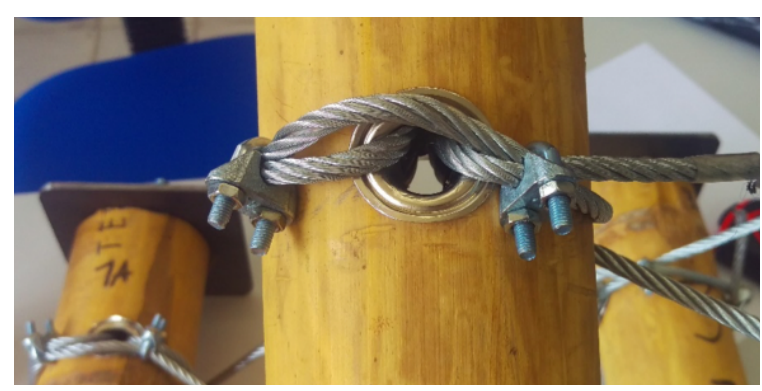

Figura 3. Acabado amarre nudo guadua.

Se ensayaron 3 probetas (ver figura 4) con una longitud de $40 \mathrm{~cm}$, diámetros y espesores como lo muestra la tabla 1 , estas probetas fueron ensayadas a tensión, sujetando los pernos y separándolos a una velocidad de $5 \mathrm{~mm} / \mathrm{min}$. 


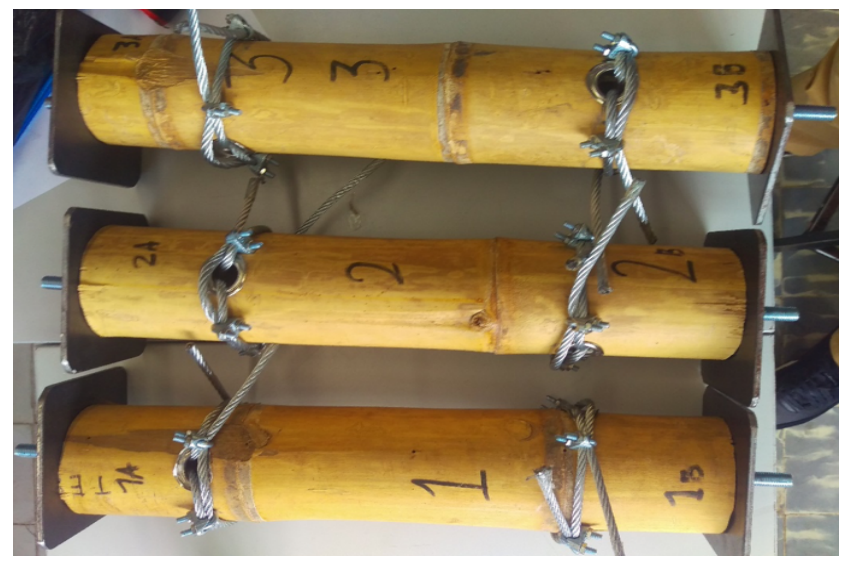

Figura 4. Probetas ensayo conexión sencilla.

Tabla 1.

Dimensiones de probetas.

\begin{tabular}{|c|c|c|c|c|}
\hline Probeta & Nudo & $\begin{array}{c}\text { Diámetro } \\
\text { promedio }(\mathbf{m m})\end{array}$ & $\begin{array}{c}\text { Espesor } \\
\text { promedio }(\mathbf{m m})\end{array}$ & Carga ultima $(\mathbf{k g})$ \\
\hline 1 & A & 91.7 & 14.5 & 2826.9 \\
\hline & B & 92.9 & 13.5 & \\
\hline 2 & A & 93.1 & 9.9 & 1133.0 \\
\hline & B & 90.7 & 9.7 & 3625.9 \\
\hline 3 & A & 90.4 & 15.2 & \\
\hline & B & 91.7 & 21.2 & \\
\hline
\end{tabular}

Las tres probetas presentaron resultados distintos, pero se muestra una pendiente similar en la ilustración carga vs desplazamiento de los tres especímenes, al llegar a la carga máxima no sufre una rotura final, sino que recibe un poco de carga más allá del desplazamiento de rotura.

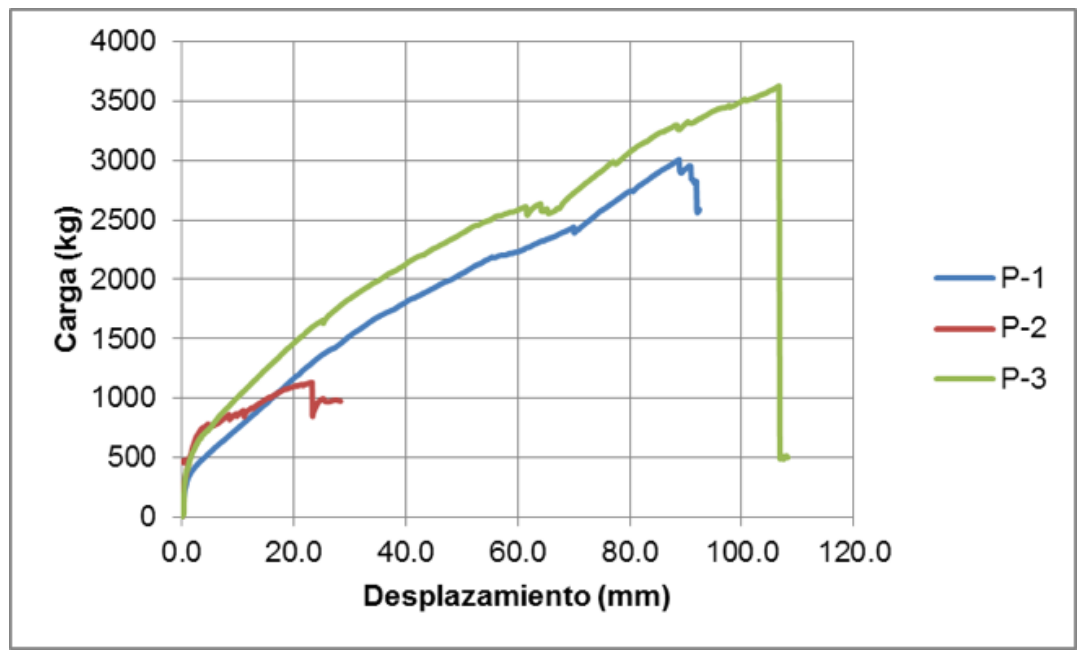

Figura 4. Grafica- Carga vs Desplazamiento.

En las fallas de las probetas 1 y 2 se observa una falla tipo bloque de cortante como la que se presenta en las conexiones metálicas (ver figura 5), lo que indica que el cable de acero realiza una presión sobre la pared superior de la perforación del culmo mientras en resto del cable lo estrangula. 


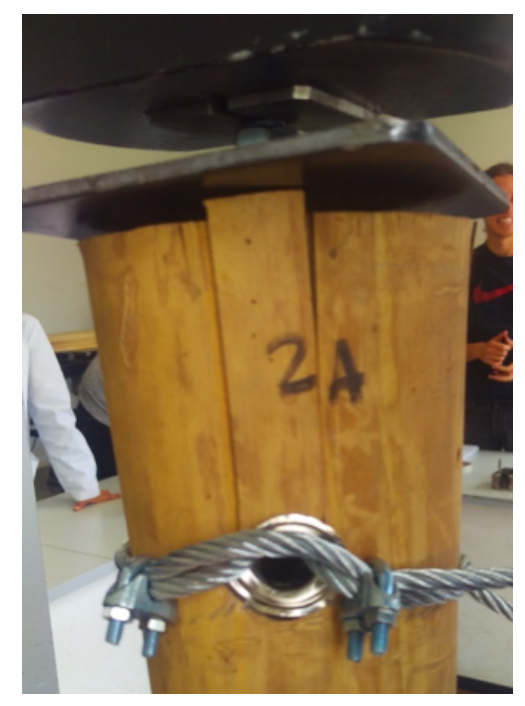

Figura 5. Falla tipo bloque de cortante.

La probeta número 3 presento una falla por tensión paralela a las fibras del culmo (ver figura 6), lo que la llevó a desarrollar mayor capacidad a la tensión (3625.9 kg).

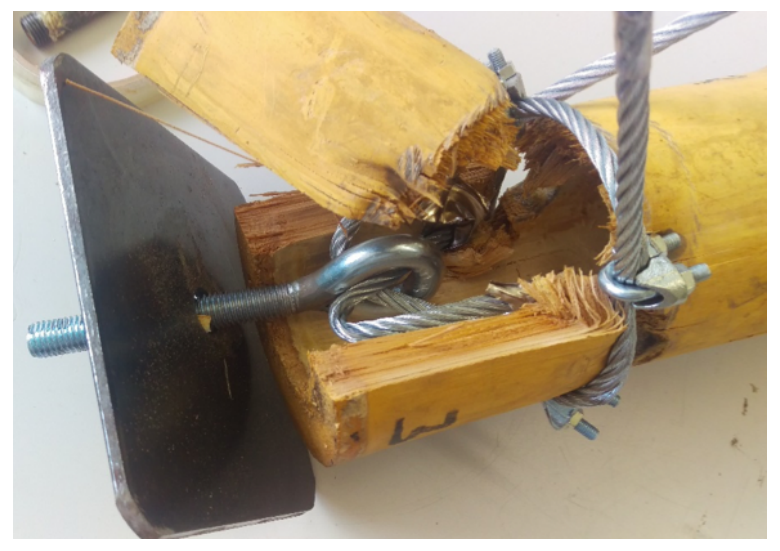

Figura 6. Falla tensión paralela a las fibras.

\subsection{DISEÑO DE NUDO METÁLICO}

El nudo metálico donde se conectan los elementos se diseñó con la carga máxima que se obtuvo en el ensayo de conexiones, teniendo en cuenta los esfuerzos generados a cortante, axial y la perforación realizada en el nudo metálico (ver figura 7), este nudo está compuesto con láminas planas de calidad ASTM- A572 Gr50 con espesor de $3 / 16 "$.

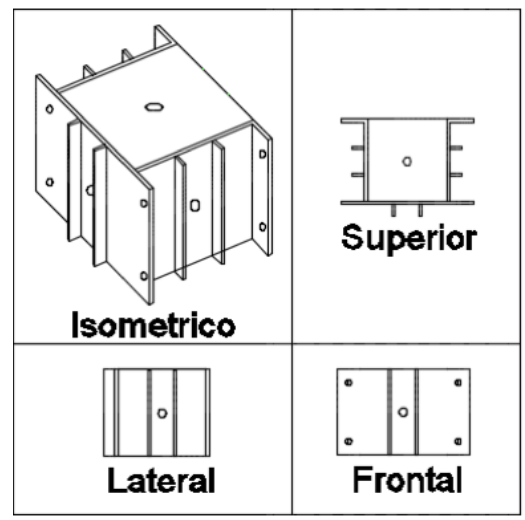

Figura 7. Esquema nudo metálico. 

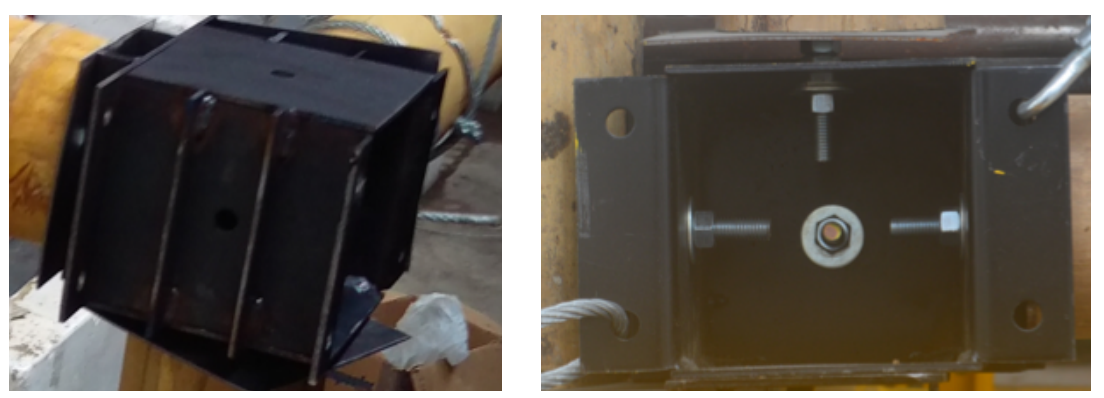

Figura 8. Fotografía nudo metálico.

Los elementos que llegaran al nudo metálico son dos vigas, una columna y una diagonal, como se muestra en la figura 8 , y se unirá entre ellos a través de pernos pasantes de 1/2" como se observa en la figura 9.

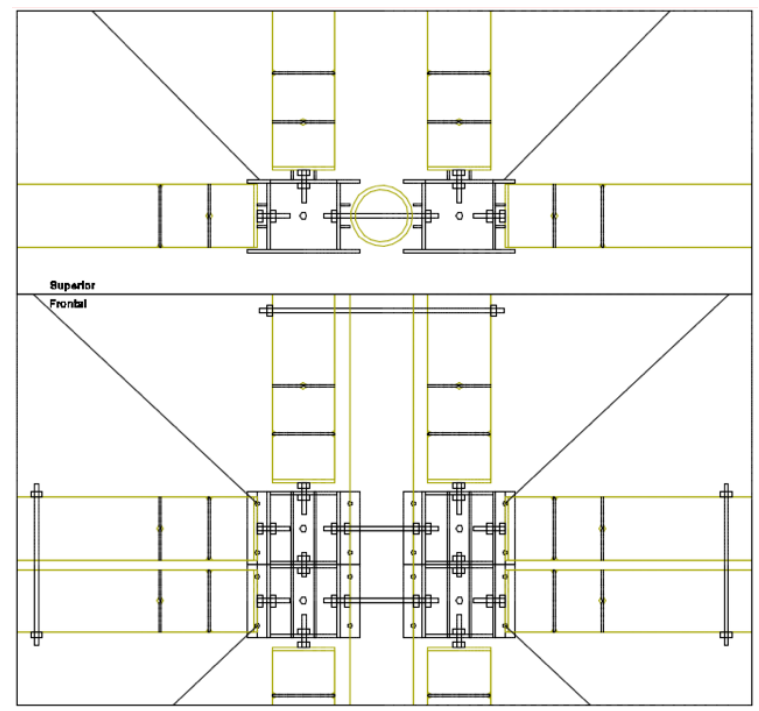

Figura 9. Nudo metálico con elementos.

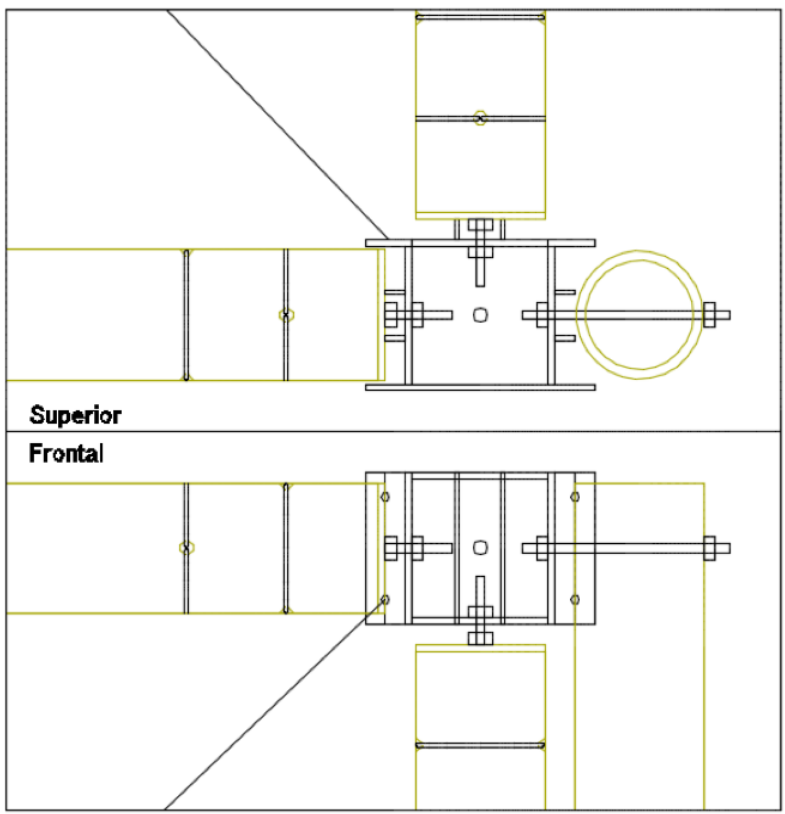

Figura 10. Unión entre módulos y nudos. 


\section{MÓDULOS ESPACIALES}

La aplicación de las conexiones descritas en el capítulo anterior será en los módulos tridimensionales de guadua rolliza, estos módulos se describen a continuación.

\subsection{CONCEPCIÓN Y DISEÑO}

Para lograr un diseño de fácil armado, económico y rápida ampliación se tomó el concepto de juegos armables para niños, donde se pueden conectar elementos ortogonales (viga y columna) a través de una conexión típica usando el concepto de tornillo para los elementos y tuerca para el nudo, la capacidad máxima de los nodos es aquella que se obtuvo de los ensayos a tensión de la conexión de los elementos en guadua.

La rigidez de cada pórtico se logra mediante cuatro tensores verticales en diagonal, uno en cada sentido y repitiendo esto en ambas caras del nudo metálico, estos tensores diagonales se ubicarán en la estructura con el mismo concepto de muros de mampostería, esto con el fin de evitar la torsión de la estructura y soportar las cargas horizontales, de igual manera la estructura tiene un diafragma rígido el cual se logra mediante tensores horizontales que unen las cuatro esquinas del módulo.

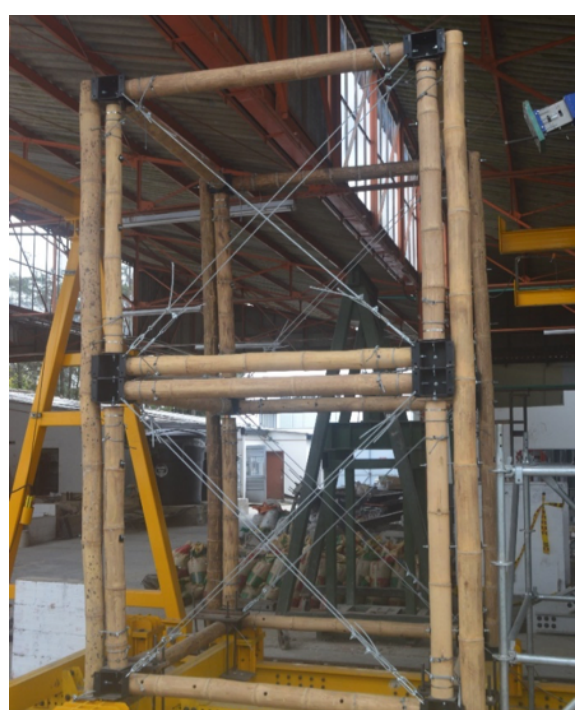

Figura 11. Módulo espacial de dos pisos.

\subsection{CONSTRUCCIÓN Y MATERIALES}

Para la construcción del módulo se usaron materiales naturales y procesados por el hombre, a continuación, se describe el tipo de material, en que elemento se usa y sus características:

Tabla 2.

Características guadua.

\begin{tabular}{|c|c|}
\hline Material & Guadua \\
\hline Elementos & Columnas y vigas \\
\hline Dimensiones & $\begin{array}{c}\text { Diámetro externo } 130 \mathrm{~mm} \\
\text { Espesor de paredes } 10 \mathrm{~mm}\end{array}$ \\
\hline Módulo de elasticidad & $15183 \mathrm{Mpa}^{1}$ \\
\hline
\end{tabular}

${ }^{1}$ (Castillo, 2017) Datos de material del mismo proveedor y en condiciones ambientales similares. 
Tabla 3.

Tabla de Características cable de acero.

\begin{tabular}{|c|c|}
\hline Material & Cable acero $6 \times 19$ \\
\hline Elementos & Tensores diagonales \\
\hline Dimensiones & $1 / 4 "$ \\
\hline Capacidad & $2400 \mathrm{~kg}$ \\
\hline
\end{tabular}

Tabla 4.

Características nudo metálico.

\begin{tabular}{|c|c|}
\hline Material & Nudo metálico A572 Gr50 \\
\hline Elementos & Nudo metálico \\
\hline Dimensiones & Espesor:3/16" \\
& Alto: $200 \mathrm{~mm}$ \\
& Ancho: $200 \mathrm{~mm}$ \\
& Fondo: $200 \mathrm{~mm}$ \\
\hline Capacidad & $5000 \mathrm{~kg}$ \\
\hline
\end{tabular}

A continuación, se describe el paso a paso del armado del módulo de prueba:

1. Se armo dos (2) cuadrantes horizontales, uno de piso segundo nivel y otro de techo primer nivel, los cuales están compuestos por cuatro (4) elementos tipo viga y cuatro (4) elementos tipo nodo.

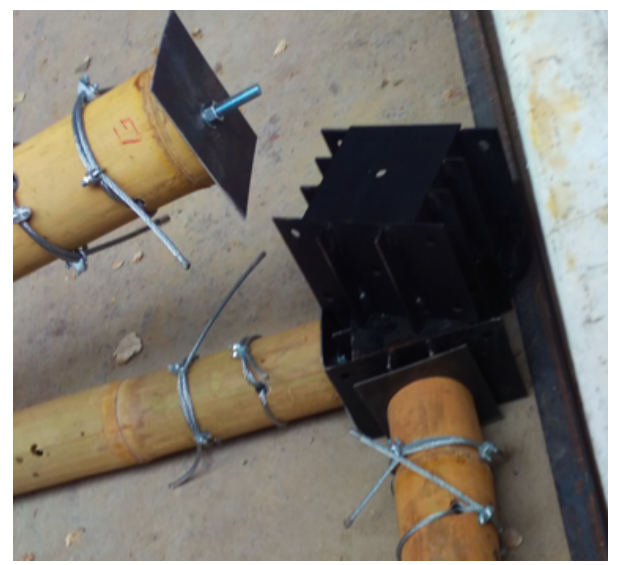

Figura 12. Unión Viga-nudo.

2. Los cuadrantes se rigidizan utilizando los tensores para generar ángulos internos de $90^{\circ}$ entre los elementos, así garantizar un diafragma horizontal.

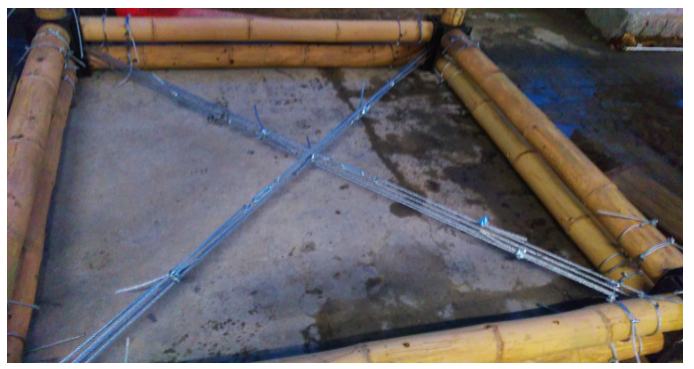

Figura 13. Diafragma de módulo. 
3. Se arma cuadrante de piso primer nivel, se izan columnas cortas y se conectan los cuadrantes armados en el paso 1.

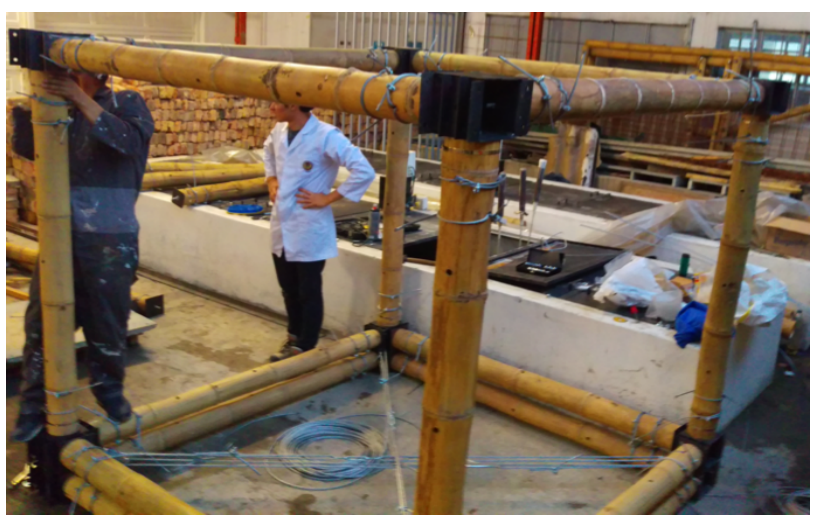

Figura 14. Diafragma de módulo.

4. Las columnas largas son izadas y se conectan a las columnas cortas del módulo de primer nivel.

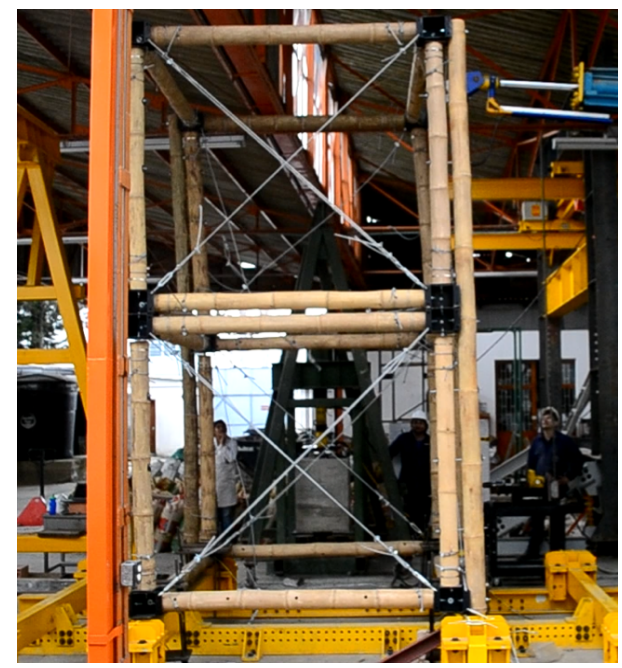

Figura 15. Verticalidad de nivel 1 y columnas de unión.

5. Se izan las columnas cortas del módulo superior y se une el techo del segundo nivel, se une las columnas largas con las columnas cortas del módulo superior.

6. Los cables de acero se conectas a los nodos y se inicia a dar verticalidad a la estructura por medio de estos cables.

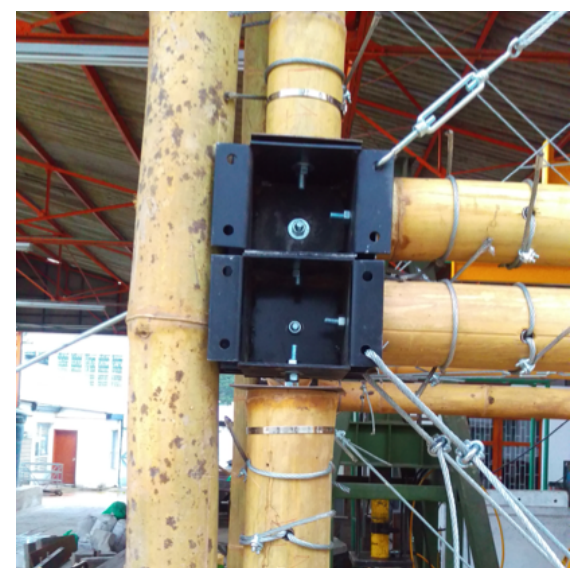

Figura 16. Verticalidad de estructura. 


\subsection{DESCRIPCIÓN DEL ENSAYO}

Se realizaron dos ejercicios, el primero aplicando la carga directamente sobre la viga superior del módulo del segundo nivel y la segunda aplicando la carga horizontal en la parte superior del módulo por medio de un elemento en acero rígido, para aplicar la carga en la parte superior de las columnas con el fin de medir el desplazamiento y concluir la capacidad de este ante cargas sísmicas, el ensayo fue realizado en el laboratorio de estructuras de la facultad de ingeniería de la Universidad Nacional de Colombia sede Bogotá.

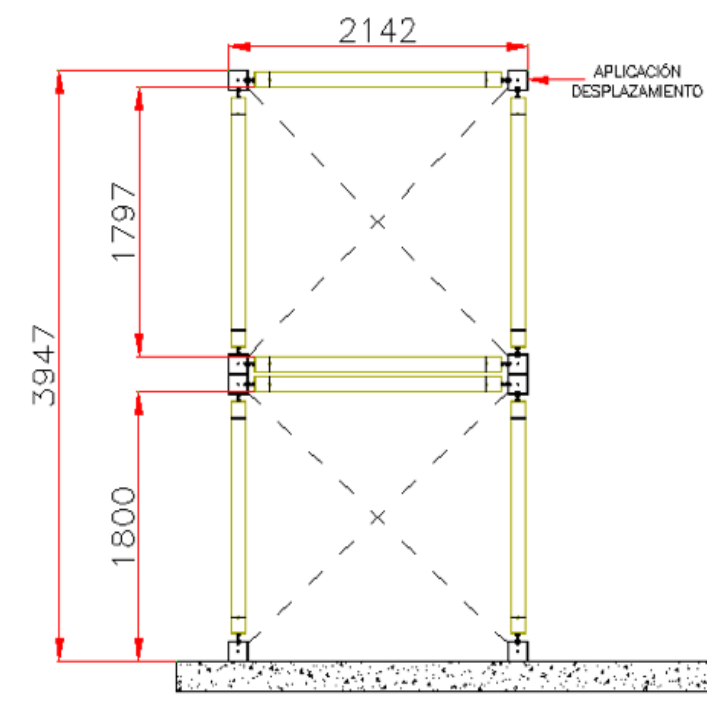

Figura 17. Esquema módulo de prueba.

El equipo usado es el actuador horizontal con desplazamiento máximo de $70 \mathrm{~cm}$ y carga de 30 toneladas con un sensor de desplazamiento Panasonic FPXC30R.

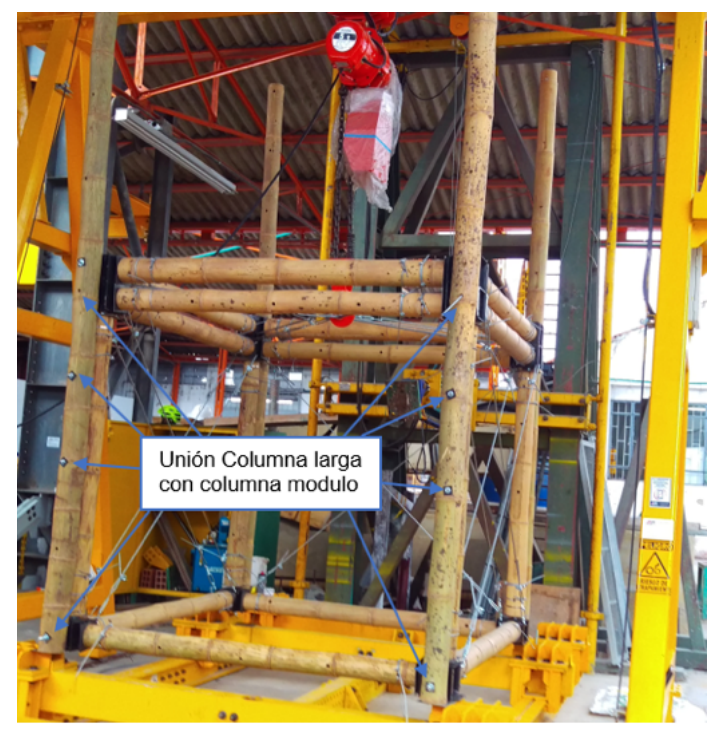

Figura 18. Imagen de prueba de laboratorio.

\section{RESULTADOS}

Los resultados obtenidos se observan en la figura 19, con una carga máxima de $1162.47 \mathrm{~kg}$ y un desplazamiento de $400 \mathrm{~mm}$ para la carga aplicada obre la viga de guadua, y una carga de $2296 \mathrm{~kg}$ y desplazamiento de $74 \mathrm{~mm}$ para la carga aplicada sobre el extremo superior de las columnas; En ambos casos la falla se presentó en las diagonales que trabajaban a tensión (figura 20). 
Tabla 5.

Aplicación de carga y resultados.

\begin{tabular}{|l|c|c|}
\hline \multicolumn{1}{|c|}{ Modo de aplicación de carga } & Carga Máxima (kg) & $\begin{array}{c}\text { Desplazamiento } \\
\text { máximo (mm) }\end{array}$ \\
\hline Sobre viga de guadua segundo nivel & 1162 & 400 \\
\hline $\begin{array}{l}\text { Con elemento rígido de acero sobre } \\
\text { parte superior columnas }\end{array}$ & 2296 & 74 \\
\hline
\end{tabular}

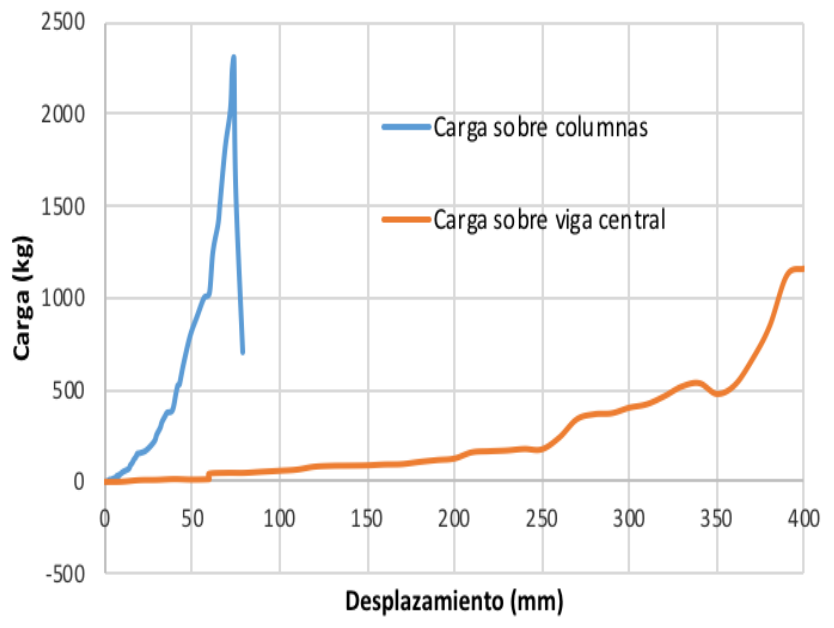

Figura 19. Gráfica- Carga vs Desplazamiento en módulo.

Se observa que los primeros desplazamientos son excesivos para una carga tan baja, esto debido a que la estructura al momento de recibir carga comienza a acomodarse, tanto los tensores como las conexiones viga nodo, por ello se observa un cambio de pendiente en la curva (carga - desplazamiento).

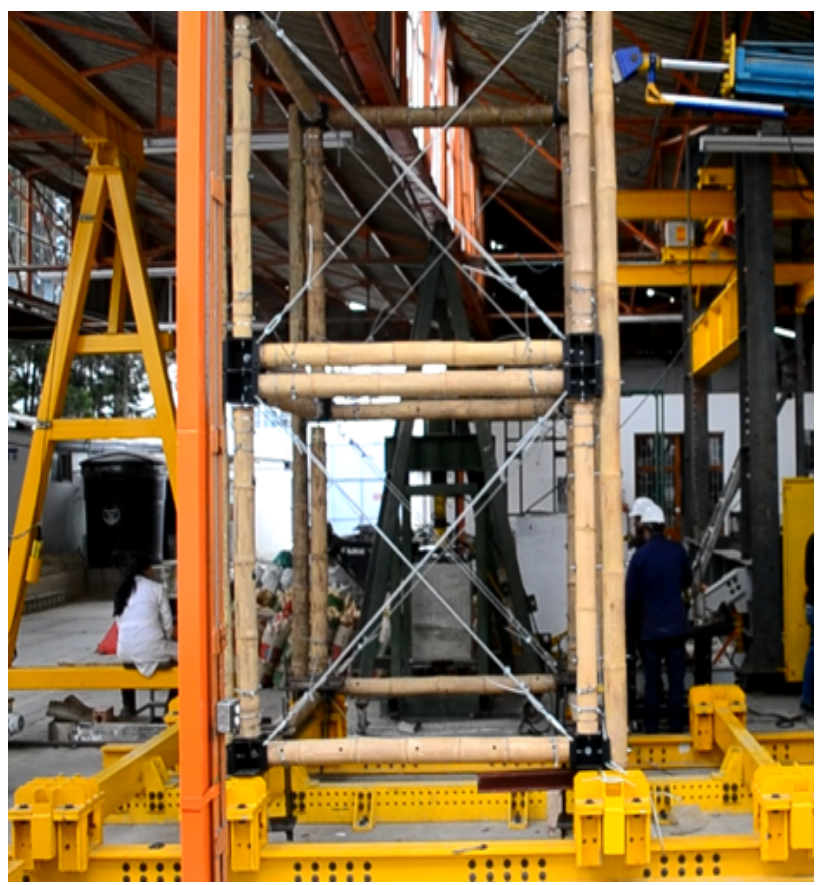

Figura 20. Fotografía del módulo después del fallo. 


\subsection{MODELO NUMÉRICO DEL MÓDULO ESPACIAL}

Se realizo la modelación en el programa computacional SAP2000 (figura 14) para revisar las cargas que actúan sobre los elementos en el ensayo al módulo, de esta modelación se encontraron las siguientes cargas:

Tabla 6.

Dimensiones de probetas.

\begin{tabular}{|l|l|}
\hline Elemento & Carga (kg) \\
\hline Dinf & 1553.66 \\
\hline Dsup & 1551.42 \\
\hline Col 1 & 977.10 \\
\hline Col 2 & 2107.40 \\
\hline
\end{tabular}

Figura 21. Esquema de modelación en SAP 2000.

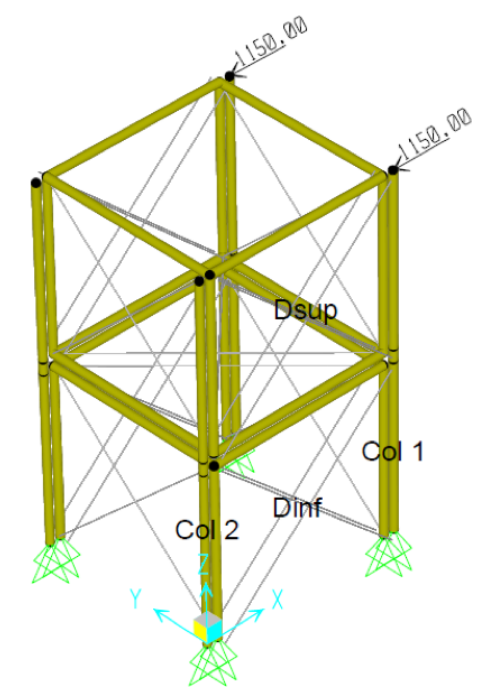

\subsection{APLICABILIDAD}

Los módulos espaciales pueden ser usados para vivienda rural de dos pisos o casas de emergencia, que dada la precariedad en algunas regiones terminan por convertirse en soluciones permanentes, a continuación, se ilustra un espacio habitacional de dos niveles usando la configuración de módulos espaciales.

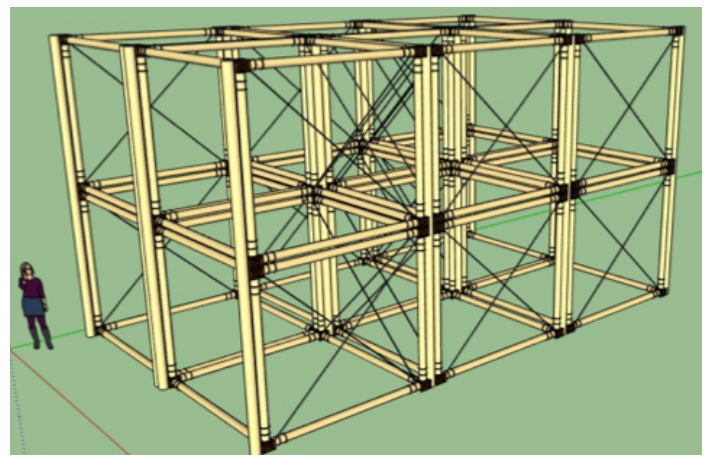

Figura 22. Isométrico estructura vivienda. 


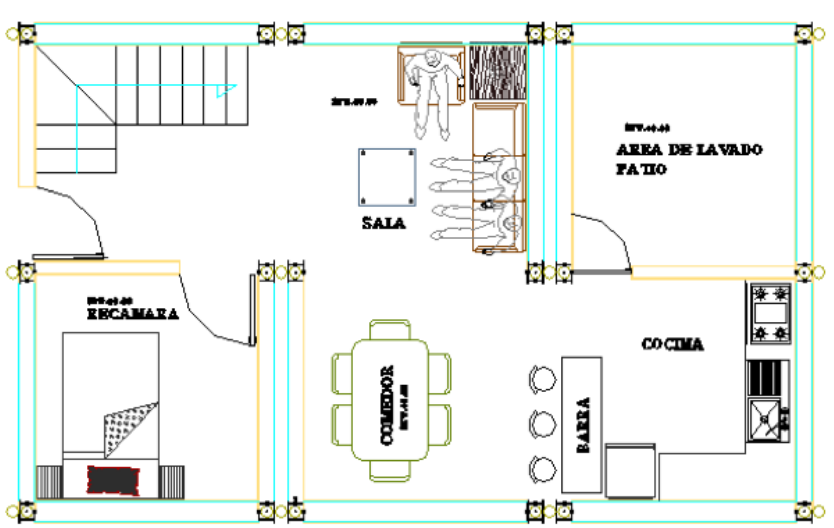

Figura 23. Planta arquitectónica primer nivel.

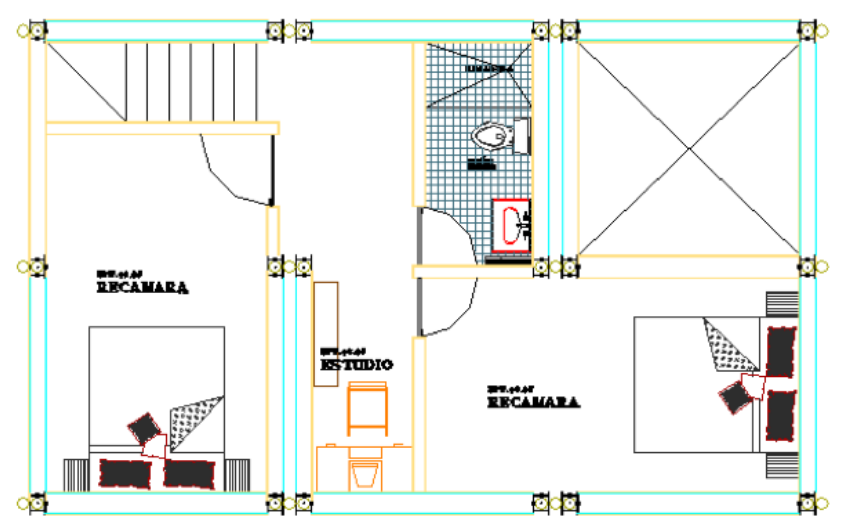

Figura 24. Planta arquitectónica segundo nivel.

\section{CONCLUSIONES}

El sistema presenta una rápida y limpia construcción, lo que facilita su uso para viviendas de emergencia y para la ampliación de estructuras existentes, el módulo de prueba se construyó en 4 días con 4 personas sin experiencia en construcción, lo que es una muestra de su facilidad.

De la modelación matemática y el ensayo realizado se identifica que los elementos que resisten la carga horizontal son los cables diagonales, como la falla se presentó en el gancho que conecta el cable con el nudo, la capacidad de la estructura se puede aumentar si se elimina el gancho del tensor y se remplaza por un ojo cerrado.

Es necesario aplicar la carga directamente sobre los extremos de las columnas, realizar una deformación previa y ajuste de los elementos, para eliminar los pequeños desplazamientos que se realizan en los elementos de la estructura por el ajuste de los cables de acero.

Para un mejor desempeño de la estructura es necesario utilizar elementos no estructurales muy ligeros, para disminuir la masa que actuara con la carga sísmica.

\section{REFERENCIAS}

Castaño, F., \& Moreno, R. D. (2004). Guadua para todos: Cultivo y aprovechamiento. Proyecto Manejo Sostenible de Bosques de Colombia. 
Sistema modular en bambú guadua con conexiones metálicas de fácil armado para vivienda rural

Echandía, C. (2000). El conflicto armado colombiano en los años noventa: cambios en las estrategias y efectos económicos. Colombia Internacional, 5.

Herrera, J., \& Takeuchi, caori. (2009). Comportamiento de pórticos en Guadua angustifolia, rigidizados mediante páneles prefabricados en bahareque. Ingeniería E Investigación, 29.

Lamus, F., Plazas, M., \& Luna, P. (2015). Resistencia de una conexión empernada solicitada a cizalladura doble paralela a la fibra para estructuras de Guadua angustifolia TT - Bolted join strength under parallel to grain double shear in Guadua angustifolia structures. Tecnura, 19(43), 52-62. https://doi.org/10.14483/udistrital. jour.tecnura.2015.1.a03

Londono, X. (1990). Aspectos sobre la distribución y la ecologia de los bambues de Colombia. Caldesia, 16, $139-153$.

Luna, P., Lozano, J., \& Takeuchi, C. (2014). Determinación experimental de valores característicos de resistencia para Guadua angustifolia. Maderas, Cinecia y Tecnologia, 16, 77-92.

Osorio, J., González, E., \& Cortés, E. (2010). La Guadua: Fundamentos para el diseño de estructuras agropecurias (1st ed.). Medellín.

Robledo, J., Muñoz, J., \& Duque, G. (1999). Al Bahareque le fue muy bien - The Bahareque * made it very well.

Sabate, P. (1998). NTP 155: Cables de acero.

Takeuchi, caori. (2004). Comportamiento estructural de la Guadua angustifolia. Uniones en guadua. Ingeniería E Investigación, 55, 3-7.

Widyowijatnoko, A. (2012). Traditional and Innovative Joints in Bamboo Construction (1st ed.).

Barnet, Y., \& Jabrane, F. (2019). Conectores de extremidades de bambú para estructuras exploracion de un sistema de incrustación en la pared intena del tallo. Campus V. XXIV. 53-66.

Castillo, C. (2017). Comportamiento del sistema estructural de proticos de columnas y paneles prefabricados (PCPP) en Guadua angustifolia ante cargas horizontales. Universidad Nacional de Colombia.

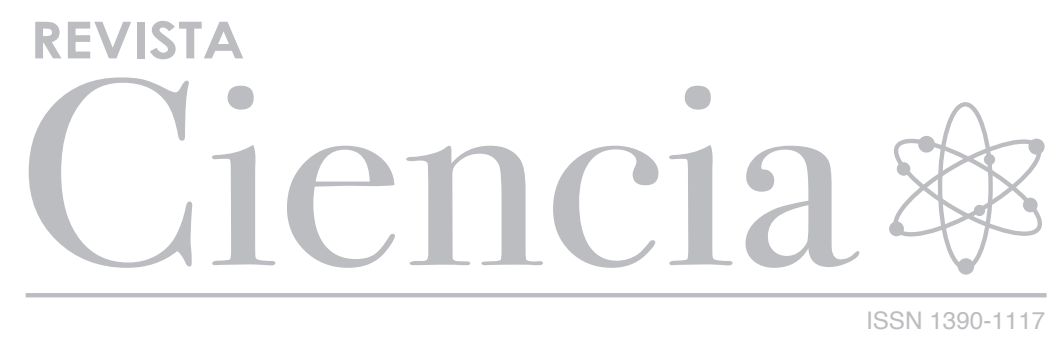

(C) Universidad de las Fuerzas Armadas ESPE Sangolquí, Ecuador.

CIENCIA - QUITO, Volumen 22, No. 1, Enero 2020

ISSN: 1390-1117, ISSN E: 2697-343X.

Revista Trimestral- 50 ejemplares 